\title{
Optimización de la Producción de Alquil Ésteres a partir de Aceite de Palma, empleando la Metodología de Superficie de Respuesta
}

\author{
Paula C. Mazo, Gloria M. Restrepo, Luis A. Ríos y Juan Miguel Marín \\ Univ. de Antioquia, Facultad de Ingeniería, Departamento Ingeniería Química, Grupo Procesos \\ Fisicoquímicos Aplicados, Calle 67 N 53-108, Medellín, Antioquia-Colombia \\ (e-mail: pcmazo@matematicas.udea.edu.co)
}

\begin{abstract}
Resumen
En el presente artículo se estudia el efecto del porcentaje másico del catalizador y la relación molar aceite:alcohol para diferentes sistemas de alcohol-catalizador, en la optimización de la producción de alquil ésteres de aceite crudo de palma. Se usó un diseño factorial $3^{2}$ y la metodología de superficie de respuesta, donde se obtuvieron las ecuaciones polinomiales cuadráticas mediante análisis de regresión múltiple. La respuesta evaluada fue el porcentaje de conversión a glicerina empleando la norma de la Sociedad Americana de Químicos de Aceites, AOCS Ca 14-56. Las dos variables afectan significativamente la conversión y el efecto de la relación molar es mayor que el de la cantidad de catalizador. Las condiciones óptimas varían para cada sistema, pero en general para los catalizadores homogéneos el porcentaje másico del catalizador es menor (1 a 2\%) que para los heterogéneos ( 5 a $6 \%$ ) y se requieren altas relaciones molares (1:12 a 1:40). Las propiedades de los combustibles obtenidos cumplen con los requerimientos de los estándares americanos para el biodiesel.
\end{abstract}

Palabras claves: biodiesel, alquil ésteres, optimización, superficie de respuesta, aceite de palma

\section{Optimization of the Alkyl Esters Production from Palm Oil using Response Surface Methodology}

\begin{abstract}
This paper studies the effect of the mass percentage of the catalyst and the molar ratio oil:alcohol for different alcohol-catalyst systems, in the optimization of alkyl esters production from crude palm oil. Factorial design $3^{2}$ and response surface methodology were used and quadratic polynomial equations by a multiple regression analysis were obtained. The evaluated answer was the percentage of conversion to glycerine using the norm of the American Oil Chemists' Society, AOCS $\mathrm{Ca}$ 14-56. The two variables significantly affect the conversion and the molar ratio effect is larger than the effect of the amount of catalyst. Optimum conditions vary for each system, but in general for the homogeneous catalysts the mass percentage of the catalyst is smaller (1 to $2 \%$ ) than for heterogeneous (5 to $6 \%$ ) and large molar ratio are required (1:12-1:40). The properties of fuel obtained are according to American standard requirements for biodiesel.
\end{abstract}

Keywords: biodiesel, alkyl esters, optimization, response surface, palm oil 


\section{INTRODUCCIÓN}

Colombia se encuentra en un período de transición en el tema de los combustibles debido a la disminución de las reservas de petróleo y a la ausencia de nuevos hallazgos; las perspectivas para la producción de fuentes energéticas viables como el biodiesel están centradas específicamente en el que se deriva del Aceite de Palma debido a que nuestro país es el quinto productor mundial y cuarto exportador de este aceite (Fedepalma, 2007). El biodiesel, obtenido vía transesterificación de aceites vegetales o grasas animales, se convierte en una alternativa atractiva, entre otras razones por su carácter biodegradable, la posibilidad de autonomía energética regional, sus bajas emisiones contaminantes y su ausencia de azufre (Agudelo et al., 2003; Ramos y Wilhelm, 2005; Ma y Hanna, 1999)

La reacción general de transesterificación de triglicéridos con metanol se muestra en la Fig.1 y se denomina metanólisis, en el caso de otros alcoholes se llama alcoholisis. Es un proceso de etapas reversibles y procede esencialmente con la mezcla de los reactivos. La presencia de un catalizador homogéneo, un ácido o una base fuerte, o heterogéneo, acelera la reacción (Marchetti et al., 2007)

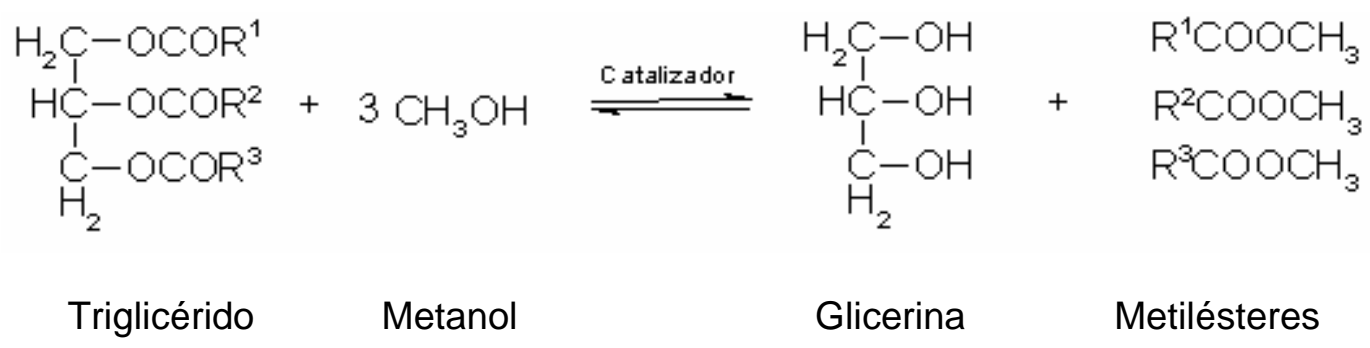

Fig. 1: Reacción general para la transesterificación de triglicéridos con metanol.

Las técnicas convencionales de síntesis para la reacción de transesterificación emplean comúnmente catalizadores homogéneos de ácidos y bases inorgánicas preferencialmente los hidróxidos y los alcóxidos de sodio y potasio. La utilización de estos catalizadores presenta desventajas técnicas y ambientales, debido a los procesos de neutralización de sus sales, los que a su vez, generan sobrecostos en la separación y purificación del producto final (Knothe et al., 2005). En los últimos años los trabajos reportados, acerca del desarrollo de catalizadores heterogéneos para la reacción de transesterificación han sido bastante extensos, destacándose de manera clara la tendencia de reemplazar estos catalizadores homogéneos (Macedo et al., 2006); esto contribuye a la eliminación de etapas adicionales al proceso y mejorar la economía del mismo. Debido a sus ventajas los catalizadores heterogéneos: pueden ser removidos del medio de reacción, pueden ser reciclados, la estructura química y en algunos casos su soporte permite orientar las reacciones hacia ciertos productos o reducir las que son indeseables, ofrecen la posibilidad de desarrollar procesos amigables con el medio ambiente, permiten ser empleadas durante largos periodos de tiempo sin perder actividad y no presentan peligros en su manejo o almacenamiento (Albis et al., 2005).

Algunos procesos de optimización para la producción de metil ésteres, empleando la metodología de superficie de respuesta, son reportados empleando diversos aceites y catalizadores (Vicente et al., 1998; Ghassan et al., 2004; Shashikant y Reaman, 2006; Vicente et al., 2007; Li et al., 2007), sin embargo no se encuentran reportes de optimización para la producción de alquil ésteres.

En el Biodiesel de Aceite de Palma (BAP), las propiedades de flujo a baja temperatura (PFBT) son desfavorables debido a su alto contenido de metilésteres saturados, constituyéndose en un problema técnico de operación debido a que la disminución de la temperatura favorece la formación de aglomerados cristalinos de las fracciones saturadas del biodiesel que pueden restringir o taponar el flujo a través de los conductos y filtros del sistema de inyección del motor. En sistemas multicomponentes como el aceite de palma, la cristalización involucra la competencia de varios procesos fisicoquímicos que dependen de la composición, los perfiles de temperatura y las fuerzas de cizalladura aplicadas (Mazzanti et al., 2005). Una de las alternativas para disminuir las propiedades de flujo a baja temperatura (PFBT) es emplear alquil ésteres, obtenidos a través de transesterificación con alcoholes ramificados y de mayor peso molecular que el metanol, que impiden la aglomeración de los mismos y la formación de cristales (Knothe et al., 2005; Benjumea et al., 2007) 
En este trabajo se pretende optimizar la producción de alquil ésteres de aceite crudo de palma, utilizando la metodología de superficie de respuesta, empleando alcoholes como el Isopropanol, Isobutanol, 2-Butanol, Isopentanol, y se evalúan las PFBT de los combustibles obtenidos.

\section{METODOLOGÍA}

La producción de los alquil ésteres se realizó utilizando el método de esterificación-transesterificación de acuerdo con lo descrito en la literatura (Keim, 1945; Kawahara y Ono, 1979; Jeromin et al., 1987; Haas et al., 2002), esta metodología consta de dos etapas descritas a continuación:

1. Pretratamiento-Esterificación: El aceite de palma es crudo sin ningún refinamiento, presenta un grado de acidez inicial (ASTM D664, 2005) de 13\%, valor que se encuentra por encima del límite de $1 \%$ requerido para realizar la transesterificación con catalizadores alcalinos (Shashikant y Reaman, 2006), razón por la cual se realiza una esterificación inicial empleando una relación molar aceite: metanol de 1:8, $2.5 \%$ de $\mathrm{H}_{2} \mathrm{SO}_{4}$, tiempo de 2 horas y sistema de agitación magnética con reflujo, después de la reacción se realiza una separación de fases, lavado y secado, el valor de acidez final para este aceite es de $1 \%$.

2. Transesterificación: El aceite obtenido en la primera etapa reacciona con los alcoholes Isopropanol, Isobutanol, 2-Butanol e Isopentanol grado analítico y con los catalizadores: metóxido de sodio, metóxido de potasio y carbonato de potasio. Los experimentos fueron conducidos a escala de laboratorio, en reactores de vidrio de $100 \mathrm{~mL}$, con sistema de reflujo, control de temperatura y agitación magnética.

Los metóxidos fueron obtenidos por reacción de metanol grado analítico con $\mathrm{Na}$ y $\mathrm{KOH}$ grado comercial. La reacción se inicia con la mezcla de los reactivos (aceite, alcohol y catalizador) y transcurre por un tiempo de 10 horas, a la temperatura cercana al punto de ebullición del alcohol respectivo. Después de transcurrido el tiempo de reacción, el sistema es lavado con agua hasta $\mathrm{pH}$ neutro para eliminar la glicerina y catalizador y luego es evaporado para extraer el agua y el alcohol en exceso.

El seguimiento del porcentaje de conversión (\%conv G) en las reacciones de transesterificación, se realizó mediante la determinación del glicerol libre, basados en la norma AOCS Ca 14-56 (1999), para aceites y grasas. Este método cuantifica la cantidad de glicerina libre obtenida, mediante la reacción con ácido peryódico cuyo exceso es titulado con una solución de tiosulfato usando una solución indicadora de almidón. El porcentaje de conversión se calculó con respecto a la glicerina teórica obtenida después de saponificar el aceite.

Se evaluaron las propiedades de los alquil ésteres obtenidos después de la optimización, para verificar que cumplan con los estándares internacionales como combustibles, tales como: gravedad API a $60^{\circ} \mathrm{F}$ (ASTM D287, 2005), punto de nube (cloud point) (ASTM D2500, 2005), punto de fluidez 0 de vertido (pour point) (ASTM D97, 2005), poder calorífico (ASTM D240, 2005), viscosidad a $40^{\circ} \mathrm{C}$ (ASTM D445, 2005), punto de inflamación $\left({ }^{\circ} \mathrm{C}\right)$ (ASTM D93, 2005) e índice de cetano (ASTM D613, 2005).

\section{Diseño de experimentos}

Para cada sistema alcohol-catalizador (12 en total), se realizó un diseño de experimentos factorial $3^{2}$ con cuatro repeticiones al centro y el análisis de los datos se realizó con la metodología de superficie de respuesta. En la Tabla 1, se reportan las variables y niveles empleados.

\section{Análisis estadístico.}

El análisis estadístico se realiza mediante un modelo de regresión múltiple y de varianza empleando el software StatGraphics 5.1, usando una ecuación polinomial de segundo orden: 


$$
z=\beta_{0}+\beta_{1} x+\beta_{2} y+\beta_{3} x^{2}+\beta_{4} y^{2}+\beta_{5} x y+\varepsilon
$$

Donde, $z$ es la variable de respuesta \%conversión de glicerina (\%conv G), $x$ es la variable independiente codificada de la cantidad de catalizador (cat), $y$ es la variable codificada correspondiente a la relación aceite:alcohol (relaa), $\beta_{0}, \beta_{1}, \beta_{2}, \beta_{3}, \beta_{4}, \beta_{5}$, son los coeficientes respectivos del intercepto, lineal, cuadrático e interacción, $\varepsilon$ es el término de error aleatorio.

Tabla 1: Variables y niveles empleados para la transesterificación

\begin{tabular}{|c|c|c|c|}
\cline { 2 - 4 } \multicolumn{1}{c|}{} & \multicolumn{3}{c|}{ Niveles } \\
\cline { 2 - 4 } \multicolumn{1}{c|}{} & -1 & 0 & 1 \\
\hline \% Hidróxido de potasio $(\mathrm{KOH})$ & 1.0 & 1.5 & 2.0 \\
\hline \% Carbonato de potasio $\left(\mathrm{K}_{2} \mathrm{CO}_{3}\right)$ & 2.0 & 4.0 & 6.0 \\
\hline \% Sodio metálico $(\mathrm{Na})$ & 0.6 & 0.8 & 1.0 \\
\hline Relación molar aceite:alcohol & $1: 15$ & $1: 20$ & $1: 25$ \\
\hline
\end{tabular}

Para el análisis del modelo se utilizó el estadístico $t$, debido a que se desconoce la varianza poblacional y el número de muestras es menor que 30, los $p$-valores mayores que 0.05 , indican que no hay relaciones estadísticamente significativas entre las variables a un nivel de confianza del 95\%.

Para determinar el punto estacionario se calcula la derivada de la superficie de respuesta con respecto a cada una de las variables codificadas y se resuelve el sistema de ecuaciones, luego se calculan los valores reales.

$$
\frac{\partial Z}{\partial y}=0 \Rightarrow y \quad \frac{\partial Z}{\partial x}=0 \Rightarrow x
$$

Para determinar si el punto estacionario corresponde a un máximo, mínimo o punto silla, se debe emplear la matriz Hessiana, la cual es:

$$
H=\left|\begin{array}{ll}
\frac{\partial^{2} z}{\partial^{2} x} & \frac{\partial^{2} z}{\partial x \partial y} \\
\frac{\partial^{2} z}{\partial y \partial x} & \frac{\partial^{2} z}{\partial^{2} y}
\end{array}\right|
$$

Y se calculan los determinantes de las submatrices (Det), dependiendo del signo obtenido se puede clasificar el punto estacionario así:

Det $\mathrm{H}_{1}<0$ y Det $\mathrm{H}_{2}>0$, el punto es un máximo

Det $\mathrm{H}_{1}>0$ y Det $\mathrm{H}_{2}>0$, el punto es silla

Det $\mathrm{H}_{1}<0$ y Det $\mathrm{H}_{2}<0$, el punto es silla

Det $\mathrm{H}_{1}>0$ y Det $\mathrm{H}_{2}<0$, el punto es mínimo

\section{RESULTADOS Y DISCUSIÓN}

\section{Valores experimentales obtenidos}

Los valores experimentales obtenidos para el \%conv G, de cada uno de los sistemas alcoholcatalizador se describen en la Tabla 2. 
Tabla 2: Diseño Factorial $3^{2}$ y repuesta para la transesterificaciçon con isopropanol, 2-butanol e isopentanol

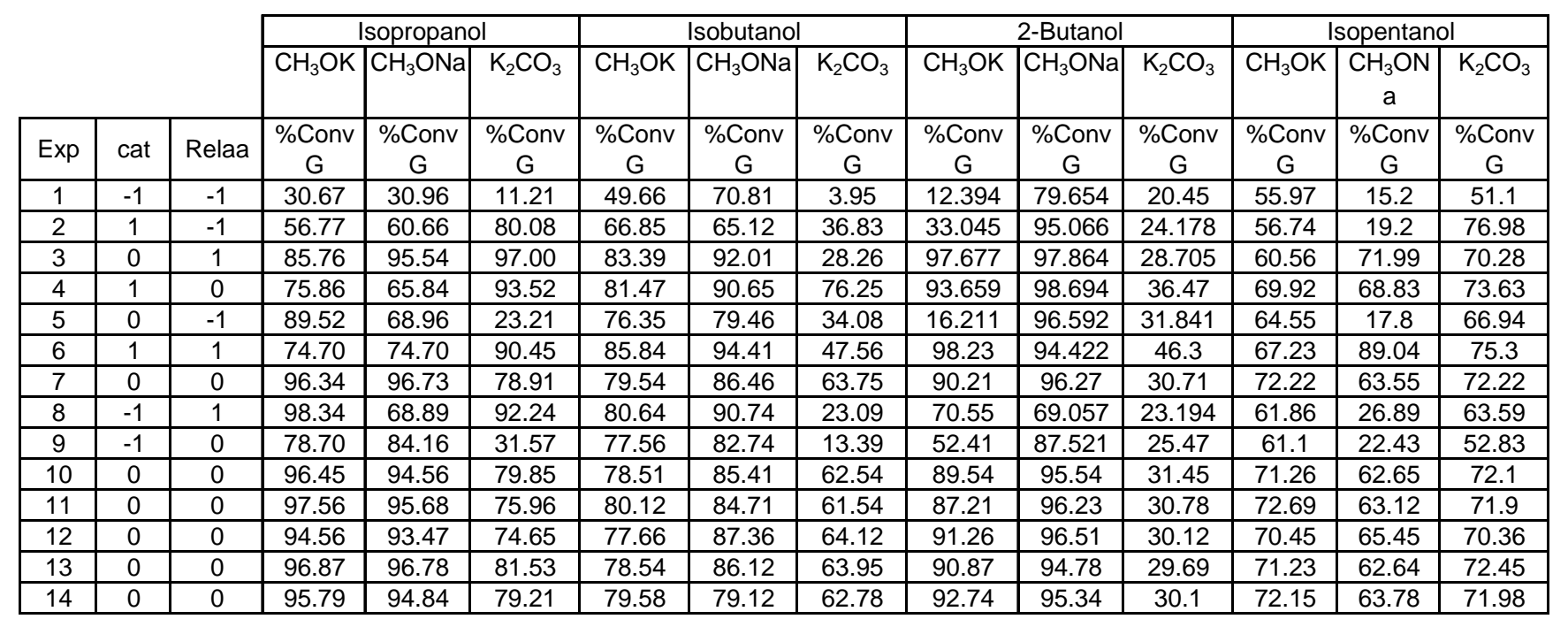

\section{Análisis de regresión múltiple}

Los valores obtenidos para los coeficientes con su respectivo $p$-valor, para los sistemas evaluados se reportan en la Tabla 3.

De acuerdo con los p-valores reportados en la Tabla 3, se puede inferir que:

- En todos los sistemas el valor de la constante es estadísticamente significativo, por lo tanto debe ser considerado en la ecuación de la superficie de respuesta.

- El término correspondiente con \% de catalizador $(X)$ no es significativo para los sistemas 1,2,5.

- El término correspondiente con la relación aceite:alcohol $(Y)$ no es significativo para los sistemas $6,8,10$.

- El término de la interacción $X^{2}$ no es significativo para los sistemas 3,4,5.

- El término de la interacción $Y^{2}$ no es significativo para 1,3,4,5,8,9,12.

- El término de la interacción $X Y$ no es significativo para el mayor número de sistemas evaluados $2,4,5,6,7,8,9,10$.

- Los modelos podrían ajustarse de acuerdo con el análisis realizado de los p-valores, pero en este trabajo se utilizarán todos los términos.

- También se aprecia, que las dos variables afectan significativamente la conversión, el efecto de la relación molar es mayor que el de la cantidad de catalizador para los sistemas 1,2,3,4,5,7,11, pero ocurre lo contrario para los sistemas 6,8,9,12 que emplean carbonato de potasio como catalizador, en el caso del sistema 10 son similares los efectos de las dos variables, esto es debido a que el anión carbonato es una base débil y reacciona muy lentamente con el alcohol, por esta razón es requerida grandes cantidades de catalizador $(>3 \%)$, para obtener resultados similares a los catalizadores homogéneos. tal como se muestra en la ecuación (4).

$$
\mathrm{K}_{2} \mathrm{CO}_{3}+\mathrm{ROH} \leftrightarrows \mathrm{RO}^{-}+\mathrm{KHCO}_{3}+\mathrm{K}^{+}
$$

En el caso del sistema 8, el 2-butoxido de sodio es un nucleófilo que presenta alto impedimento ésterico dificultando la reacción, por esta razón se requieren altas concentraciones de catalizador, pero un aumento del catalizador homogéneo puede interferir y disminuir el rendimiento por la posible formación de jabón (Kucek et al., 2007). 
El alcohol se usa en exceso para obtener altos rendimientos en la formación de alquil ésteres como lo exponen Fangrui y Milford (1999), Lee (1995); Lotero et al. (2006); Majan et al. (2007) y se confirma con los resultados obtenidos.

Tabla 3: Análisis de regresión múltiple

\begin{tabular}{|c|c|c|c|c|c|c|c|}
\hline \multirow[b]{2}{*}{$\mathrm{N}^{\circ}$} & \multirow[b]{2}{*}{ Sistema } & \multicolumn{6}{|c|}{ Superficie de Respuesta } \\
\hline & & Constante & $x$ & $\bar{Y}$ & $\mathrm{X}^{2}$ & $\mathrm{Y}^{2}$ & $\overline{X Y}$ \\
\hline \multirow{2}{*}{1} & Isopropanol- $\mathrm{CH}_{3} \mathrm{OK}$ & 96,6778 & $-0,065$ & 13,6402 & $-20,6467$ & $-10,2872$ & $-12,4322$ \\
\hline & p-valor & 0,0000 & 0,9872 & 0,0085 & 0,0069 & 0,11 & 0,0326 \\
\hline \multirow{2}{*}{2} & Isopropanol- $\mathrm{CH}_{3} \mathrm{ONa}$ & 95,7079 & 2,8653 & 13,0918 & $-21,8025$ & $-14,554$ & $-5,9725$ \\
\hline & p-valor & 0,0000 & 0,3765 & 0,0027 & 0,0012 & 0,0114 & 0,1497 \\
\hline \multirow{2}{*}{3} & Isopropanol- $\mathrm{K}_{2} \mathrm{CO}_{3}$ & 75,5051 & 21,5072 & 27,532 & $-4,4197$ & $-6,8612$ & $-17,663$ \\
\hline & p-valor & 0,0000 & 0,0014 & 0,0003 & 0,52 & 0,3267 & 0,0127 \\
\hline \multirow{2}{*}{4} & Isobutanol- $\mathrm{CH}_{3} \mathrm{OK}$ & 80,1261 & 4,3842 & 9,5017 & $-4,0156$ & $-3,6601$ & $-2,9968$ \\
\hline & $\mathrm{p}$-valor & 0,0000 & 0,0612 & 0,0015 & 0,2079 & 0,2471 & 0,2591 \\
\hline \multirow{2}{*}{5} & Isobutanol- $\mathrm{CH}_{3} \mathrm{ONa}$ & 85,7218 & 0,9817 & 10,295 & $-1,6021$ & $-2,5621$ & 2,34 \\
\hline & p-valor & 0,0000 & 0,6012 & 0,0005 & 0,5587 & 0,9577 & 0,3205 \\
\hline \multirow{2}{*}{6} & Isobutanol- $\mathrm{K}_{2} \mathrm{CO}_{3}$ & 61,3509 & 20,035 & 4,0083 & $-11,2435$ & $-24,8935$ & $-2,1025$ \\
\hline & p-valor & 0,0000 & 0,0008 & 0,3234 & 0,0771 & 0,002 & 0,6642 \\
\hline \multirow{2}{*}{7} & 2-butanol- $\mathrm{CH}_{3} \mathrm{OK}$ & 88,6719 & 14,93 & 34,1345 & $-10,7381$ & $-26,8286$ & 1,7573 \\
\hline & p-valor & 0,0000 & 0,0007 & 0,0000 & 0,0307 & 0,0002 & 0,6244 \\
\hline \multirow{2}{*}{8} & 2-butanol- $\mathrm{CH}_{3} \mathrm{ONa}$ & 96,9557 & 8,6583 & $-1,6615$ & $-7,3802$ & $-3,2597$ & 2,4883 \\
\hline & $\mathrm{p}$-valor & 0,0000 & 0,0005 & 0,3184 & 0,0117 & 0,1894 & 0,2295 \\
\hline \multirow{2}{*}{9} & 2-butanol- $\mathrm{K}_{2} \mathrm{CO}_{3}$ & 30,7382 & 6,3057 & 3,6217 & 4,8445 & $-1,2549$ & $-0,5579$ \\
\hline & p-valor & 0,0000 & 0,0016 & 0,0283 & 0,0194 & 0,5426 & 0,7845 \\
\hline \multirow{2}{*}{10} & Isopentanol- $\mathrm{CH}_{3} \mathrm{OK}$ & 71,19 & 2,4933 & 2,065 & $-4,25$ & $-7,205$ & 1,15 \\
\hline & p-valor & 0,0000 & 0,082 & 0,1382 & 0,0482 & 0,0042 & 0,4754 \\
\hline \multirow{2}{*}{11} & Isopentanol- $\mathrm{CH}_{3} \mathrm{ONa}$ & 62,2859 & 18,7583 & 22,62 & $-12,9185$ & $-13,6535$ & 14,5375 \\
\hline & $\mathrm{p}$-valor & 0,0000 & 0,0000 & 0,0000 & 0,0026 & 0,0019 & 0,0004 \\
\hline \multirow{2}{*}{12} & Isopentanol- $\mathrm{K}_{2} \mathrm{CO}_{3}$ & 71,0424 & 9,7317 & 2,3583 & $-5,4344$ & $-0,0544$ & $-3,5425$ \\
\hline & p-valor & 0,0000 & 0,0000 & 0,0286 & 0,0029 & 0,9673 & 0,0114 \\
\hline
\end{tabular}

Del análisis de varianza (ver Tabla 4) se aprecia que los modelos de regresión son significativos para todos los sistemas ya que los p-valores obtenidos son menores que 0,05 , y de acuerdo a los coeficientes de determinación $R^{2}$ y $R_{a j}{ }^{2}$ el modelo explica bien la variabilidad presente en los datos, estos son mayores que 0,7 (Gutiérrez y De la Vara, 2004) y los sistemas no presentan multicolinealidad. El sistema que presenta el error estándar de estimación y la media del error absoluto de mayor magnitud es el 3 , debido al alto impedimento estérico que presenta el isopropóxido de potasio y a la dificultad de su formación con el carbonato por las razones antes expuestas, los otros sistemas presentan valores pequeños de acuerdo con la escala de medición de la variable de respuesta (0-100\%).

En la Tabla 5 se reportan los valores obtenidos para el porcentaje de conversión a glicerina en los puntos estacionarios encontrados para cada uno de los sistemas evaluados donde se efectuaron 3 repeticiones. 
Tabla 4: Análisis de varianza

\begin{tabular}{|c|c|c|c|c|c|c|c|c|c|c|}
\hline Sist & $\begin{array}{l}\text { Fuente de } \\
\text { variación }\end{array}$ & $\begin{array}{l}\text { Suma de } \\
\text { cuadrado }\end{array}$ & $\begin{array}{l}\mathrm{G} \\
\mathrm{L}\end{array}$ & $\begin{array}{l}\text { Cuadrad } \\
\text { o medio }\end{array}$ & $\mathrm{F}_{\mathrm{o}}$ & $\mathrm{p}$-valor & $\overline{R^{2}}$ & $\mathrm{R}_{\mathrm{aj}}{ }^{2}$ & $\begin{array}{c}\text { Error } \\
\text { estándar de } \\
\text { estimación }\end{array}$ & $\begin{array}{c}\text { Media } \\
\text { del error } \\
\text { absoluto }\end{array}$ \\
\hline & Modelo & 4165,82 & 5 & 833,164 & 8,98 & 0,0039 & 0,849 & 0,754 & 9,6334 & 4,9610 \\
\hline 1 & $\begin{array}{l}\text { Residual } \\
\text { Total }\end{array}$ & $\begin{array}{l}742,418 \\
4908,24\end{array}$ & $\begin{array}{c}8 \\
13\end{array}$ & 92,8023 & & & & & & \\
\hline \multirow[b]{3}{*}{2} & Modelo & 4482,95 & 5 & 896,589 & 15,96 & 0,0006 & 0,909 & 0,852 & 7,4958 & 4,0486 \\
\hline & Residual & 449,496 & 8 & 56,1871 & & & & & & \\
\hline & Total & 4932,44 & 13 & & & & & & & \\
\hline \multirow[b]{3}{*}{3} & Modelo & 8886,36 & 5 & 1777,27 & 14,54 & 0,0008 & 0,901 & 0,839 & 11,0563 & 5,9872 \\
\hline & Residual & 977,927 & 8 & 122,241 & & & & & & \\
\hline & Total & $9,864,29$ & 13 & & & & & & & \\
\hline \multirow[b]{3}{*}{4} & Modelo & 836,146 & 5 & 167,229 & 6,87 & 0,009 & 0,811 & 0,693 & 4,9342 & 2,681 \\
\hline & Residual & 194,773 & 8 & 24,3466 & & & & & & \\
\hline & Total & 1030,92 & 13 & & & & & & & \\
\hline \multirow[b]{3}{*}{5} & Modelo & 706,639 & 5 & 141,328 & 7,24 & 0,0077 & 0,819 & 0,706 & 4,4195 & 2,4468 \\
\hline & Residual & 156,256 & 8 & 19,5319 & & & & & & \\
\hline & Total & 862,895 & 13 & & & & & & & \\
\hline \multirow{3}{*}{6} & Modelo & 5880,25 & 5 & 1176,05 & 13,51 & 0,001 & 0,894 & 0,828 & 9,33126 & 5,0573 \\
\hline & Residual & 696,58 & 8 & 87,0725 & & & & & & \\
\hline & Total & 6576,83 & 13 & & & & & & & \\
\hline \multirow[b]{3}{*}{7} & Modelo & 12027 & 5 & 2405,41 & 50,47 & $.0,000$ & 0,969 & 0,950 & 6,90337 & 3,8929 \\
\hline & Residual & 381,252 & 8 & & & & & & & \\
\hline & Total & 12408,3 & 13 & & & & & & & \\
\hline \multirow[b]{3}{*}{8} & Modelo & 783,041 & 5 & 156,608 & 10,7 & 0,0022 & 0,870 & 0,789 & 3,8253 & 2,1495 \\
\hline & Residual & 117,064 & 8 & 14,633 & & & & & & \\
\hline & Total & 900,105 & 13 & & & & & & & \\
\hline \multirow[b]{3}{*}{9} & Modelo & 419,612 & 5 & 83,9223 & 7,61 & 0,0066 & 0,826 & 0,717 & 3,32154 & 1,8183 \\
\hline & Residual & 88,2609 & 8 & 11,0326 & & & & & & \\
\hline & Total & 507,872 & 13 & & & & & & & \\
\hline \multirow[b]{3}{*}{10} & Modelo & 395,578 & 5 & 79,1155 & 8,39 & 0,0048 & 0,840 & 0,740 & 3,0717 & 1,7438 \\
\hline & Residual & 75,4827 & 8 & 9,43534 & & & & & & \\
\hline & Total & 471,06 & 13 & & & & & & & \\
\hline \multirow[b]{3}{*}{11} & Modelo & 7741,88 & 5 & 1548,38 & 60,75 & $.0,000$ & 0,974 & 0,958 & 5,0484 & 2,7116 \\
\hline & Residual & 203,897 & 8 & 25,4871 & & & & & & \\
\hline & Total & 7945,78 & 13 & & & & & & & \\
\hline \multirow[b]{3}{*}{12} & Modelo & 753,91 & 5 & 150,782 & 32,08 & $.0,000$ & 0,952 & 0,923 & 2,168 & 1,4563 \\
\hline & Residual & 37,6017 & & & & & & & & \\
\hline & Total & 791,512 & & & & & & & & \\
\hline
\end{tabular}

Con el análisis de regresión múltiple es posible predecir el porcentaje de conversión a partir de unas condiciones de reacción (concentración del catalizador y relación molar aceite-alcohol), en este caso se evaluaron los puntos estacionarios obtenidos, este es un punto de partida para la síntesis de 
estos biocombustibles a escala piloto e industrial. Los valores predichos para cada uno de los puntos estacionarios confirman que los modelos obtenidos son adecuados.

Los porcentajes de conversión menores que el 90\% obtenidos experimentalmente fueron los sistemas: Isobutanol- $\mathrm{CH}_{3} \mathrm{OK}$. Isobutanol- $\mathrm{K}_{2} \mathrm{CO}_{3}$.2-butanol- $\mathrm{K}_{2} \mathrm{CO}_{3}$. Isoamílico- $\mathrm{CH}_{3} \mathrm{OK}$. Isoamilico$\mathrm{K}_{2} \mathrm{CO}_{3}$ esto es debido a que las reacciones se dificultan porque la reacción de obtención del metóxido de potasio como catalizador forma agua que puede hidrolizar el triglicérido formar ácidos grasos libres y luego sales que impiden el ataque nucleofílico del metóxido por impedimento estérico. Además el agua puede solvatar el ión y desactivarlo disminuyendo su reactividad y aumentando su consumo.

Tabla 5: Puntos estacionarios diseños experimentales

\begin{tabular}{|l|c|c|c|c|c|c|}
\hline \multicolumn{1}{|c|}{ Sistema } & \multicolumn{2}{|c|}{$\begin{array}{c}\text { Punto } \\
\text { estacionario } \\
\text { Valores sin } \\
\text { codificar }\end{array}$} & Tipo & $\begin{array}{c}\text { \%conv } \\
\text { teórico }\end{array}$ & $\begin{array}{c}\text { \%conv } \\
\text { Experimental } \\
\text { promedio }\end{array}$ & $\begin{array}{c}\text { Desviación } \\
\text { estándar }\end{array}$ \\
\hline & \%Cat & Relaa & & & & \\
\hline Isopropanol- $\mathrm{CH}_{3} \mathrm{OK}$ & 1.377 & 24.058 & Máximo & 97.23 & 94.03 & 2.13 \\
\hline Isopropanol- $\mathrm{CH}_{3} \mathrm{ONa}$ & 0.801 & 22.245 & Máximo & 98.65 & 92.74 & 7.49 \\
\hline Isopropanol- $\mathrm{K}_{2} \mathrm{CO}_{3}$ & 6.005 & 23.580 & Silla & 96.14 & 95.26 & 1.05 \\
\hline Isobutanol- $\mathrm{CH}_{3} \mathrm{OK}$ & 1.305 & 32.543 & Máximo & 79.10 & 74.02 & 9.18 \\
\hline Isobutanol- $\mathrm{CH}_{3} \mathrm{ONa}$ & 1.332 & 36.122 & Máximo & 103.63 & 94.93 & 4.30 \\
\hline Isobutanol- $\mathrm{K}_{2} \mathrm{CO}_{3}$ & 5.774 & 20.216 & Máximo & 70.32 & 64.49 & 7.91 \\
\hline 2-butanol- $\mathrm{CH}_{3} \mathrm{OK}$ & 1.875 & 23.304 & Máximo & 105.54 & 97.07 & 1.48 \\
\hline 2-butanol- $\mathrm{CH}_{3} \mathrm{ONa}$ & 0.916 & 19.835 & Máximo & 99.48 & 96.74 & 2.04 \\
\hline 2-butanol- $\mathrm{K}_{2} \mathrm{CO}_{3}$ & 0.751 & 11.539 & Silla & 55.36 & 57.48 & 7.26 \\
\hline Isoamilico- $\mathrm{CH}_{3} \mathrm{OK}$ & 1.658 & 20.843 & Máximo & 71.76 & 71.69 & 0.98 \\
\hline Isoamilico- $\mathrm{CH}_{3} \mathrm{ONa}$ & 7.404 & 28.672 & Máximo & 97.86 & 96.70 & 1.25 \\
\hline Isoamilico- $\mathrm{K}_{2} \mathrm{CO}_{3}$ & 0.928 & 23.890 & Silla & 75.88 & 73.59 & 4.52 \\
\hline
\end{tabular}

\section{Evaluación de las propiedades}

En la Tabla 6 se reportan los valores obtenidos para las principales propiedades de los alquilésteres. Con referencia a las propiedades de los alquilésteres se observa que tienen puntos de nube y fluidez similares y que en general poseen menores propiedades de flujo en frío que los metilésteres. El punto de nube más bajo corresponde a los isopropilésteres con los cuales se logra una reducción de $10{ }^{\circ} \mathrm{C}$ con respecto al valor de dicha propiedad para los metilésteres. Las propiedades de los combustibles obtenidos cumplen con los requerimientos de los estándares americanos para el biodiesel, por lo cual pueden usarse solos o en mezcla.

Tabla 6: Propiedades de alquilésteres del aceite de palma (a, Tomado de Dishington, 2007)

\begin{tabular}{|c|c|c|c|c|c|}
\hline \multicolumn{7}{|c|}{ Alcoholes } \\
\hline Propiedad & Metiléster ${ }^{\text {a }}$ & Isoamiléster & Isobutiléster & Isopropiléster & 2-butiléster \\
\hline Gravedad api A 60 ${ }^{\circ} \mathrm{F}$ & 30.21 & 32.3 & 32.0 & 32.3 & 32.1 \\
\hline Punto de nube $\left({ }^{\circ} \mathrm{C}\right)$ & 18 & 8.0 & 7.5 & 6.0 & 7.5 \\
\hline Punto de fluidez $\left({ }^{\circ} \mathrm{C}\right)$ & 12 & -9.0 & -3.0 & -3.0 & -6.0 \\
\hline $\begin{array}{c}\text { Poder calorífico } \\
\text { (btu/lb) }\end{array}$ & 17380 & 17607 & 17511 & 17457 & 17465 \\
\hline $\begin{array}{c}\text { Viscosidad a 40 } \\
\text { (cst) }\end{array}$ & 4.490 & 5.856 & 5.586 & 5.021 & 6.186 \\
\hline $\begin{array}{c}\text { Punto de inflamación } \\
\left({ }^{\circ} \mathrm{C}\right)\end{array}$ & 30 & 99.0 & 120.0 & 114.0 & 88.0 \\
\hline Número de cetano & 48 & 51.88 & 51.42 & 51.36 & 51.18 \\
\hline
\end{tabular}




\section{CONCLUSIONES}

Se evaluó el efecto del porcentaje en peso del catalizador y la relación molar aceite:alcohol para 12 sistemas de alcohol-catalizador, donde se obtuvieron ecuaciones polinomiales cuadráticas por análisis de regresión múltiple. Las dos variables afectan significativamente la conversión, el efecto de la relación molar es mayor para la mayoría de los sistemas, la cantidad de catalizador es más significativo para los sistemas que utilizan carbonato de potasio, debido a su baja reactividad. Las condiciones óptimas varían para cada sistema, pero en general para los catalizadores homogéneos la cantidad es menor (1-2\%) que para los heterogéneos (5-6\%) y se requieren altas relaciones molares aceite:alcohol (1:12-1:40). Las propiedades de los combustibles obtenidos cumplen con los requerimientos de los estándares americanos para el biodiesel, los alquil ésteres de palma, presentan menores propiedades de flujo en frío que los metil ésteres, por lo cual son apropiados para su uso en regiones con bajas temperaturas.

\section{AGRADECIMIENTOS}

Al grupo de Procesos Fisicoquímicos Aplicados por permitir el desarrollo de este trabajo de investigación, Conciencias por la financiación y a la Universidad de Antioquia.

\section{REFERENCIAS}

Agudelo, J.R., P. Benjumea y L. Corredor; Biodiesel de aceite de palma: una alternativa para el desarrollo del país y para la autosuficiencia energética nacional, Rev. Fac. Ing. Univ. Antioquia: 28, 50-61 (2003).

Albis, A., J. Parra y F. Sánchez; Transesterificación del aceite de palma con metanol por catálisis heterogénea. Revista ingeniería e investigación: 25 (2), 71-77 (2005).

AOCS Ca 14-56. American Oil Chemists'Society. Monoglycerides and Free Glycerol. Champaign, Illinois (1999).

ASTM D287, American Society for Testing and Materials, Standard Test Method for API Gravity of Crude Petroleum and Petroleum Products (Hydrometer Method). Philadelphia, Estados US (2005).

ASTM D2500, American Society for Testing and Materials. Standard test method of cloud point of petroleum products. Philadelphia, US (2005).

ASTM D97. American Society for Testing and Materials. Standard test method for pour point of petroleum products. Philadelphia, US (2005).

ASTM D445. American Society for Testing and Materials. Standard test method for kinematic viscosity of transparent and opaque liquids (and the calculation of dynamic viscosity). Philadelphia, US (2005).

ASTM D240. American Society for Testing and Materials. Standard Test Method of Hydrocarbon Fuels by Bomb Calorimeter. Philadelphia, US (2005).

ASTM D93. American Society for Testing and Materials. Standard Test Methods for Flash-Point by Pensky-Martens Closed Cup Tester. Philadelphia, US (2005).

ASTM D613. American Society for Testing and Materials. Standard Test Method for Cetane Number of Diesel Oil. Philadelphia, US (2005).

ASTM D664. American Society for Testing and Materials. Standard Test Method for Acid Number of Petroleum Products by Potentiometric Titatrion. Philadelphia, US (2005).

Benjumea, P., J.R. Agudelo y L.A. Ríos; Propiedades de flujo a baja temperatura del biodiesel de aceite de palma. Rev. Fac. Ing. Univ. Antioquia: 42, 94-104 (2007). 
Dishington, J.; Foro biocombustibles: Potencia de Colombia. Fedepalma. Cartagena de Indias, 7 de septiembre 2007. http://www.minagricultura.gov.co/archivos/foro_cartagena_biodiesel_de_palma_ sept_7-2007.pdf._Acceso: 21 de enero (2008).

Fangrui, M y A. Milford; Biodiesel production: a review. Bioresource Technology: 70 1-15 (1999).

Haas, M. et al. Production of FAME from Acid Oil. a By-product of Vegetable Oil Refining. JAOCS 80 1 97-102 (2003).

Fedepalma., Estructura del mercado mundial de aceites y grasas (2007), http://www.fedepalma.org/ eco_mercado_mundial.shtm. (Acceso: 9 de Enero 2008)

Ghassan, T., A. Mohamad y A. Mohammad; Experimental study on evaluation and optimization of conversion of waste animal fat into biodiesel. Energy Conversion and Management: 45, 2697-2711 (2004).

Gutierrez, H. y R. De la Vara; Análisis y diseño de experimentos. 1ª edición. 430. Mc Graw Hill. Mexico (2004).

Haas, M., S. Bloomer y K. Scott; Process for the Production of Fatty Acid Alkyl Esters. US Patent No. 6.399.800, 4 de junio (2002).

Jeromin, L., E. Peukert y G. Wollmann; Process for the Pre-Esterification of Free Fatty Acids in Fats and Oils. US Patent No. 4.698.186, 6 de octubre (1987).

Kawahara, Y y T. Ono; Process for Producing Lower Alcohol Esters of Fatty Acids. US Patent No. 4.164.506, 14 de agosto (1979).

Keim, G.; Treating Fats and Fatty Oils. US Patent No. 2.383.601, 28 de agosto (1945).

Knothe, G., J. Krahl y J. Van Gerpen; The biodiesel Handbook, $1^{\mathrm{a}}$ edición, 34-69. AOCS Press, Champaign, Illinois (2005)

Kucek, T., M. Aparecida y C. Oliveira; Ethanolysis of refined soybean oil assisted by sodium and potassium hydroxides. J. Amer. Oil Chem. Soc.: 84, 385-392 (2007).

Lee, I.; Use of branched- Chain Ester to Reduce the Crystallization Temperature of Biodiesel. JAOCS:72 10 1155-1160 (1995).

Li, W., W. Du y D. Liu; Optimization of whole cell-catalyzed methanolysis of soybean oil for biodiesel production using response surface methodology. Journal of Molecular Catalysis B: Enzymatic: 45, 122-127 (2007).

Lotero, E. y otros cinco autores; The Catalysis of Biodiesel Synthesis, Catalysis: 19, 41-83 (2006).

Ma, F. y M. Hanna; Biodiesel production: a review. Biores. Technol.: 70, 1-15 (1999)

Macedo, C., F. Abreu y A. Tavares; A. New heterogeneous metal-oxides based catalyst for vegetable oil trans-esterification. J. Braz. Chem. Soc.: 17 (7) 1291-1296 (2006).

Majan, S., S. Konar y D. Boocock; Variables Affecting the Production of Standard Biodiesel. J. Amer. Oil Chem. Soc.: 84,189-195 (2007)

Marchetti, J., V.U. Miguel y A.F. Errazu; Possible methods for biodiesel production. Renewable and Sustainable Energy Reviews: 11, 1300-1311 (2007). 
Mazzanti, G., A. Marangoni y S. Idziak; Modelling phase transitions during the crystallization of a multicomponent fat under shear, Physical Review E: 71, 041607 (2005).

Ramos, L.P. y H.M. Wilhelm; Current status of biodiesel development in Brazil. Appl. Biochem. Biotechnol.: 121-124, 807-820 (2005)

Shashikant, G. y H. Raheman; Process optimization for biodiesel production from mahua (Madhuca indica) oil using response surface methodology. Bioresource Technology: 97 379-384 (2006).

Vicente, G., A. Coteron, M. Martinez y J. Aracil; Application of the factorial design of experiments and response surface methodology to optimize biodiesel production. Industrial Crops and Products: 8, 2935 (1998).

Vicente, G., M. Martinez y J. Aracil; Optimisation of integrated biodiesel production. Part I. A study of the biodiesel purity and yield. Bioresource Technology: 98 1724-1733 (2007). 
